\title{
Enhanced cohesive zone model to predict delamination behaviour of carbon/epoxy laminated curved beams
}

David Ranz

Departamento de Diseño y Fabricación, Universidad de Zaragoza, Zaragoza, Spain dranz@unizar.es

https://orcid.org/0000-0001-8451-660X

Jesús Cuartero

Departamento de Ingeniería Mecánica, Universidad de Zaragoza, Zaragoza, Spain https://orcid.org/0000-0002-6100-7412

Luis Castejón

Departamento de Ingeniería Mecánica, Universidad de Zaragoza, Zaragoza, Spain https://orcid.org/0000-0002-9007-1560

Ramón Miralbes

Departamento de Diseño y Fabricación, Universidad de Zaragoza, Zaragoza, Spain https://orcid.org/0000-0002-9702-9314

David Valladares

Departamento de Ingeniería Mecánica, Universidad de Zaragoza, Zaragoza, Spain https://orcid.org/0000-0002-6046-4558 


\title{
Enhanced cohesive zone model to predict delamination behaviour of carbon/epoxy laminated curved beams
}

\author{
This paper proposes an enhanced Cohesive Zone Model (CZM) for the prediction \\ of delamination in curved beams of epoxy carbon laminates. This model \\ improves the conventional CZM, taking into account the fiber-bridging \\ phenomenon and the variation of the element size among the thickness in the \\ curved zone. \\ The advantages of the enhanced model are underlined when results obtained from \\ the numerical simulations of a four-point-bending test in compliance with ASTM \\ D6415 standard are compared with the corresponding experimental results. The \\ prediction of the post-failure behaviour obtained with this model is closer to that \\ obtained experimentally than with the conventional model.
}

Keywords: Interlaminar tensile strength, Cohesive elements, Delamination, Fiber-bridging, Four-point-bending, Laminated curved beam

\section{Introduction}

Currently, technological advances in the manufacturing processes of composite materials have increased the integration of functions in applications for different sectors, such as aeronautics, maritime industry, energy or civil construction [1]. This fact involves components with complex geometries, which present zones with small bending radius, which are commonly present in a wide range of engineering structures. The failure of these components is due to the poor Inter-Laminar Tensile Strength (ILTS) properties of these curved zones, leading to delamination between layers [2], [3]. Thus, the prediction of the ILTS value and its post-failure evolution is a key factor when approaching the development of efficient designs. Numerical methodologies to predict ILTS in curved beams are proposed in several studies, such as those carried out by Raju [2], Avalon [4], Martin [5], Ross [6], Gozluklu [7], Kim [8], Ju [9] and Nguyen [10]. Although, different numerical methodologies to predict the onset and evolution of 
delamination are currently implemented in the packages of finite elements, Cohesive Zone Model (CZM) [2], [7], [11]-[14] has been used in the present study. Alfano [15] stated that the bilinear law provides the best performance between the different constitutive laws for the traction-separation curve of the cohesive elements, regarding to the precision of the results and the computational cost.

That bilinear law will rule delamination evolution and is defined by the following characteristics [13], [16]-[18]: First, there is an initial elastic part having a high interface stiffness $(\mathrm{K})$, that is maintained as far as the maximum interlaminar strength is reached (To). After such peak, a softening behavior takes place until the stress is equal to zero. The area below the force-displacement curve is equal to the interlaminar fracture strength (Gc). Whenever a cohesive element reaches the maximum interlaminar strength failure initiates, and when area below the curve equals Gc cohesive element fails and delamination propagates.

As Gozluklu [7], Nguyen [10] and Ranz [19] demonstrated, Cohesive Zone Model (CZM) including bilinear law applied to curved zones provides accurate predictions of maximum force values at failure and stiffness. However, it offers null information [10], imprecise [7] or shows some discrepancy [19] with experimental results regarding post-failure behavior.

This study aims to improve this weakness presented by the bilinear model, in order to obtain a better approximation to the post-failure behavior of the experimental tests, specifically in the curved areas. To reach this goal, two new considerations are proposed which are omitted in the bilinear model.

On the one hand, the existence of the "fiber-bridging" phenomenon [11], [20][23] or friction between the delaminated plies in Mode II [24], which is a typical composite materials phenomenon, is not contemplated by the previous model. This 
phenomenon of "fiber-bridging", shown in Figure 1, is due to the presence of fibers that act as bridges between adjacent delaminated layers. In short, this phenomenon gives to the degraded interface a certain resistance against the delamination advance. This effect is mainly related to unidirectional laminates and has an impact on the energy release rate Gc, which increases as the crack grows until reaching a stable value. With this in mind, not only is the delamination evolution a consequence of the energy released by the damage occurred at the crack advance point, but it is also influenced by the subsequent contribution due to fiber-bridging.

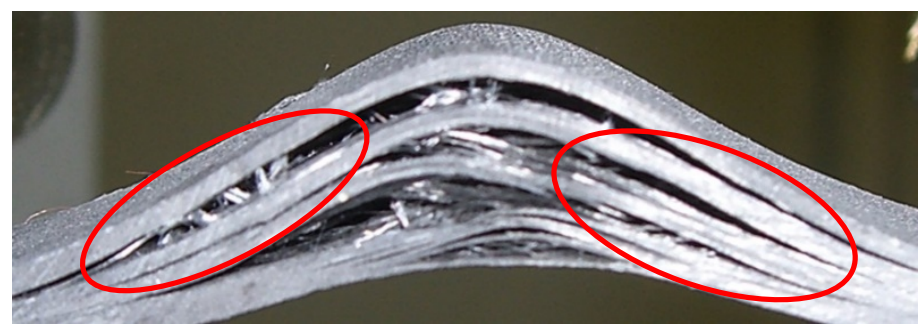

Figure 1. Fiber-bridging effect.

On the other hand, when curved areas are modeled, a strategy that follows a regular pattern is applied [7], [10], [19]. This strategy gives rise to a type of mesh that causes the cohesive elements between the different layers of the curved area to increase in size as the thickness of the specimen does. For large thicknesses, this kind of mesh can change significantly the number of elements needed for modelling the characteristic length of the cohesive zone, see Table 2, resulting in problems throughout the calculation process and inaccuracies in the expected results [11], [25]-[27].

There are previous approaches to this phenomenon using step-wise bilinear laws. However, this is only a part of our enhanced constitutive model for curved beams (CB-CZM), since this step-wise bilinear law (including fiber bridging behaviour) is adjusted considering the radial location of cohesive elements. Therefore, the novelty of our contribution underlies in the development of softening laws, which involves both fiber-bridging and radial location of cohesive elements. 
Taking into account these new considerations, an enhanced model for curved beams is developed, which has been called CB-CZM. Finally, the predicted results by CB-CZM of ILTS and post-failure evolution are correlated with the conventional CZM predicted behaviour [19] and experimental results presented by Ranz et al [28] for unidirectional (UD) 4,8,12 carbon/epoxy layered laminated curved test coupons.

\section{Curved Beam - Cohesive Zone Model description (CB-CZM)}

\subsection{Inclusion of the "fiber-bridging" phenomenon}

The conventional CZM only considers the damage phenomena occurring at the tip of the crack, related to the microstructural failure, but does not consider other effects that occur along the crack, such as the "fiber-bridging" phenomenon or frictional effects between delaminated layers (Figure 2 - left). "fiber-bridging" and frictional effects are mainly related to failure modes I and II, respectively. This process occurs in a large size area comparable to the crack size, which limits the application of analytical methods and requires the use of numerical methods for the resolution of resulting equations [21], such as the one used in this paper. If this typical phenomenon of fiber-reinforced materials and to a greater extent of unidirectional laminates [16] is not considered, then low correlation in the post-failure behavior between experimental results and numerical ones will be incurred. When this phenomenon is taken into account, the law governing the delamination evolution is expected to show a peak of stress at low displacements, which corresponds to what happens at the crack tip. Moreover it is believed to bring about a long maintained stress in the back of the crack [13], regarding to the "fiberbridging" phenomenon. This phenomenon is formally represented as a law of damage evolution divided into two segments, as shown in Figure 2 (right). 

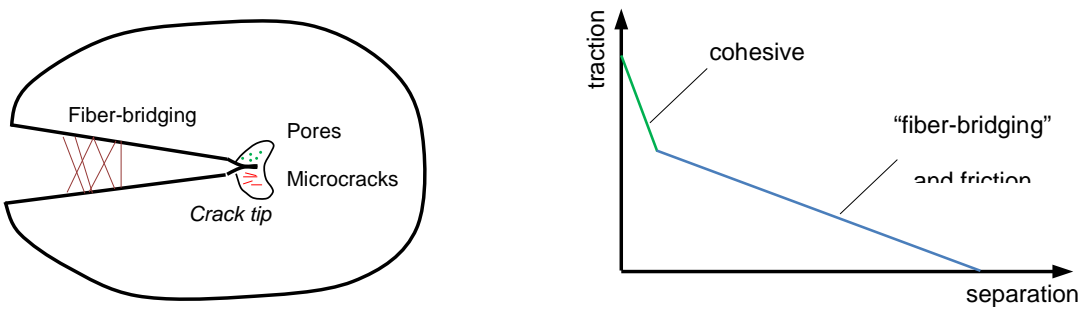

Figure 2. "Fiber-bridging” formation (left). Traction-separation mixed law of cohesivebridging (right).

Several authors [20]-[22] have worked on different methodologies aimed to numerically reproduce this phenomenon in the post-failure behavior of these materials. However, all of them have in common that there will be a differentiation in the fiberbridging contribution to the final cohesive law. On the one hand, for small separations a mechanism of brittle damage will take place, and later on, for large separations the damage will evolve withstanding low-stress levels. This approach allows achieving results closer to those obtained experimentally.

The contribution of each one of these phenomena will be defined by the superposition of two linear laws, as shown in Figure 3.

$$
\begin{array}{ll}
T_{1}^{o}=n T^{o} ; & T_{2}^{o}=(1-n) T^{o} \\
G_{1}=m G_{C} ; & G_{2}=(1-m) G_{C}
\end{array} \mid \text { con } 0 \leq n ; m \geq 1
$$

Where subscripts $\mathrm{i}=1$ and 2 denote cohesive and fiber-bridging contribution, respectively; $n$ and $m$ are the ratios of interface strength $\left(T_{0}\right)$ and energy release rate $\left(G_{C}\right)$, respectively. These rates are involved in the two laws that intervene in this softening model. In the following manner:

$$
\begin{gathered}
T^{o}=T_{1}^{o}+T_{2}^{o} \\
G_{C}=G_{1}+G_{2}
\end{gathered}
$$




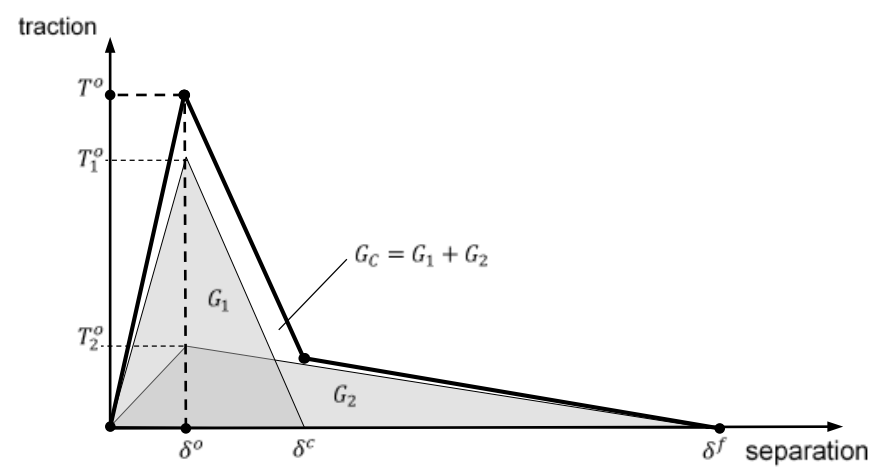

Figure 3. General constitutive law of enhanced model.

As in the bilinear model, the evolution of the damage for this multilineal model in mixed-mode situations is ruled according to the model proposed by Benzeggagh and Kenane [29], which relates the energies released for each of the modes throughout the delamination process.

The selected damage evolution law describes how the interface stiffness is degraded once the damage initiation criterion has been reached. Variable D represents the global damage to which the material is subjected and includes the combined effects of all the active mechanisms. At the beginning, when the interface is not damaged, it has a value of 0 and evolves to 1, when the interface is completely damaged, following the cohesive law used. The stress components in line with the traction-separation model are affected by the damage, as expressed in the following equations:

$$
\begin{aligned}
T_{n} & =\left\{\begin{array}{c}
(1-D) \bar{T}_{n} ; \quad \bar{T}_{n} \geq 0 \\
\bar{T}_{n} ;(\text { no damage under compression })
\end{array}\right. \\
T_{s} & =(1-D) \bar{T}_{s} \\
T_{t} & =(1-D) \bar{T}_{t} ; \quad T_{t}=0 \text { for two }- \text { dimensional models }
\end{aligned}
$$

Where subscripts n, s and t denotes normal, shear and tear directions, respectively. If this softening law is governed by two different phenomena, the damage 
evolution variable, $\mathrm{D}$, will be defined in the following way, as a ratio between the different displacement jumps:

$$
\begin{array}{lc}
D=0 ; & \text { si } \delta_{m}^{\max }<\delta_{m}^{o} \\
D=\left(\delta_{m}^{o}-\delta_{m}^{\max }\right) \frac{\left(\delta_{m}^{c}-\delta_{m}^{o}\right)}{\delta_{m}^{\max }\left(\delta_{m}^{o}-\delta_{m}^{c}\right)} ; & \text { si } \delta_{m}^{o}<\delta_{m}^{\max }<\delta_{m}^{c}
\end{array}
$$

The graphic form that represents the constitutive model of zone with a multilinear degradation model for the mixed mode is shown in Figure 4. This model shows how the fracture energy $\left(G_{c}\right)$ evolves for mixed modes between normal mode (mode I) and shear mode (mode II and III).
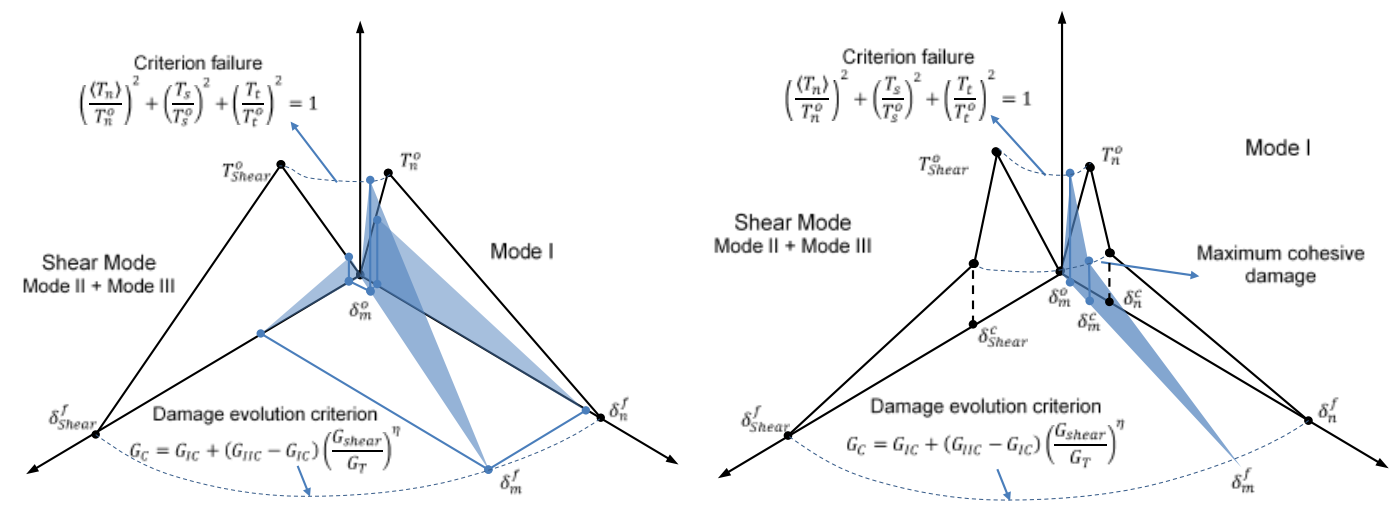

Figure 4. General constitutive law for mixed modes between mode I and pure shear mode: left) conventional [19]; right) enhanced model.

Parameters $\mathrm{n}$ and $\mathrm{m}$ shown in eq (1), which are the ratios of interface strength and energy release rate, respectively, are adjusted by testing. Experimental results obtained from the test samples including 4, 8 and 12 layers, were used to reach this goal. An accurate adjustment of the behavior law in the interface is achieved by applying this methodology. What is more, neither of the material properties had to be modified, nor any additional modeling effort had to be performed. Only the adjustment of $m$ and $n$ parameters had to be carried out. These parameters are necessary for the 
differentiation of the delamination response to in two stages. The first one governed by cohesive behavior and the second by the phenomenon of "fiber-bridging".

Table 1 gathers de $m$ y $n$ values for the laws governing failure modes I and II, while Figure 4 shows the softening laws for each of these modes (blue), including the contribution of the cohesive law (red) and the "fiber- bridging" phenomenon (green).

"Fiber-bridging" substantially contributes in terms of energy to mode I.

However, "fiber-bridging" effect is practically negligible for mode II. This contribution of "fiber-bridging" phenomenon to each of the failure mode was previously collected by Li [23], [30] in his studies.

Table 1. Relational parameters between cohesive contribution and "fiber-bridging".

\begin{tabular}{lcc}
\hline & Mode I & Mode II \\
\hline \hline $\mathrm{m}$ & 0.35 & 0.85 \\
\hline \hline $\mathrm{n}$ & 0.85 & 0.95 \\
\hline
\end{tabular}
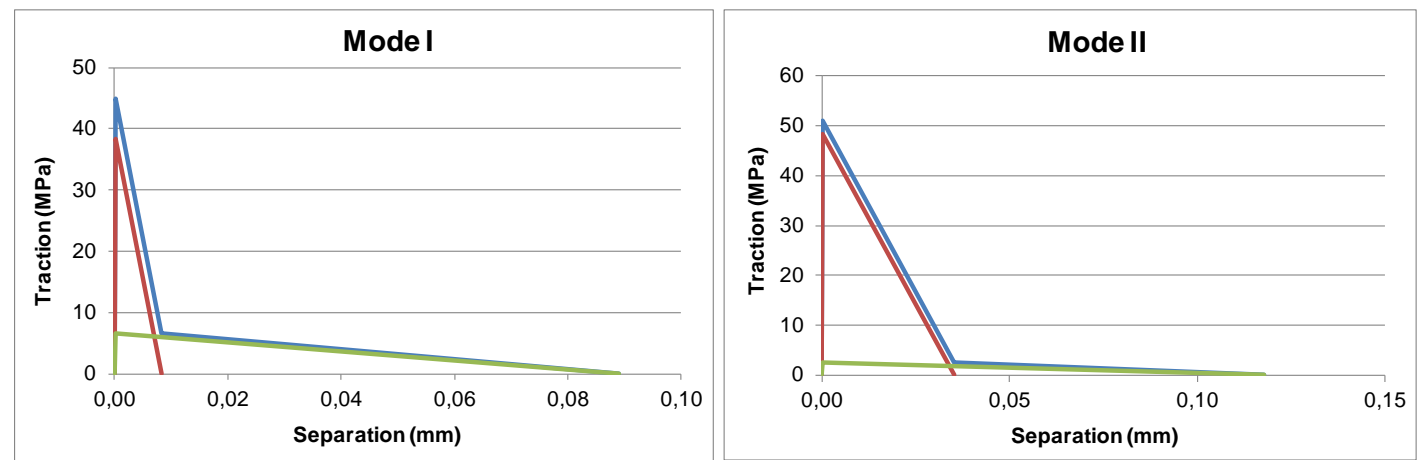

Figure 5. Softening laws for mode I and mode II in the enhanced model.

ABAQUS [31] allows us to introduce this law in two different ways for the generation of numerical models. First, through a tabular function, which jointly defines the two laws of damage evolution as a function of separation in cohesive elements, affecting each modeled phenomenon. Second, by means of superimposing independently the two laws by the duplication of cohesive elements. 
During this work, both the tabular method and the duplication of elements method were tested. However, using ABAQUS software, tabular functions were needing providing a lot of points in order to describe the zone were the element starts failing and in spite of it a lot of convergence troubles were raised. Whereas element duplication provided a better behavior in terms of numerical convergence and confidence results. For this reason element duplication method has been chosen for the development of the numerical models.

\subsection{Adjustment of the radial location effect of cohesive elements}

The typical finite element modelization applied to the study of the delamination in curved laminates is a regular one. In this kind of analysis the behavior at the ply level has to be analyzed. However, the use of a regular model leads to the appearance of very important differences in the size of the cohesive elements, between those near the inner and the outer plies. In fact, the characteristic length of the cohesive zone becomes discretized by a smaller number of elements in the outermost plies of the curved zone. Additionally, the greater the thickness of the laminate, the higher difference in the element length according to its location.

Table 2 shows, for laminates of 4, 8 and 12 layers, how the cohesive element length varies according to the element location through the laminate thickness. It can be observed how the number of elements applied in the characteristic length modelization is influenced by the element location through the laminate thickness. Figures included in Table 2 are related to the application of 24 elements to model the curved zone. It can be noted that for a 12-layer laminate the discretization of the inner interface presents more than twice as many elements as the outermost interface, using a single element to model the characteristic length. 
Nevertheless, the different studies do not provide clarity on the minimum number of elements considered to be used in the cohesive zone. Moës and Belytschko [32] suggest using more than 10 elements, Falk and others [33] use 2 to 5 elements in their simulations, whereas Camanho and Davila [16] consider that 3 elements are sufficient to predict the delamination evolution due to mode I, which is related to the characteristic length of the most restrictive cohesive zone.

Table 2. Element length $\left(l_{e}\right)$ and number of elements $\left(N_{e}\right)$ in the characteristic length according to its radial location.

\begin{tabular}{ccccccc}
\hline \multirow{2}{*}{$\begin{array}{c}\text { Cohesive } \\
\text { element location }\end{array}$} & \multicolumn{2}{c}{ 4 layers } & \multicolumn{2}{c}{ 8 layers } & \multicolumn{2}{c}{ 12 layers } \\
\cline { 2 - 7 } & $\mathrm{I}_{\mathbf{e}}$ & $\mathbf{N}_{\mathbf{e}}$ & $\mathrm{I}_{\mathbf{e}}$ & $\mathbf{N}_{\mathbf{e}}$ & $\mathrm{I}_{\mathbf{e}}$ & $\mathbf{N}_{\mathbf{e}}$ \\
\hline \hline Inner & 0.482 & 2.5 & 0.474 & 2.6 & 0.473 & 2.6 \\
\hline \hline Medium & 0.545 & 2.2 & 0.638 & 1.9 & 0.743 & 1.6 \\
\hline \hline Outer & 0.608 & 2.0 & 0.803 & 1.5 & 1.013 & 1.2 \\
\hline
\end{tabular}

Furthermore, critical energy release rate is modified artificially to rectify this discretization geometrical effect. Turon [14] uses a similar technique by increasing artificially the interface strength. By means of this method discretizations with greater length of cohesive element are obtained, which provide good numerical predictions.

A better behavior of the models in terms of numerical convergence is achieved by applying this adjustment of the energy, related to the element position along the radius. Moreover, results in terms of force-displacement graphs are not influenced [19] by the small implemented modifications of the critical energy release rate.

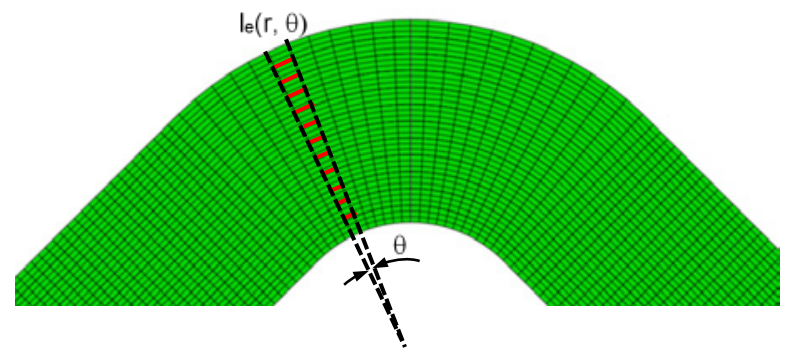

Figure 6. Radial location correction of the cohesive element. 
Aimed to carry out this correction, the geometric relationship between the element length $\left(\mathrm{l}_{\mathrm{e}}\right)$ and its radial position $\left(\mathrm{r}_{\mathrm{e}}\right)$ is established:

$$
l_{e}=r_{e} \sin \left(\frac{\theta}{2}\right)
$$

Where $\theta$ is the covered angle. Moreover, taking into consideration the equation stated by Turon (6), the characteristic length of the cohesive zone can be determined. Combining eq (5) and (6) $G_{c}$ appears as a function of the radial location of the cohesive element:

$$
\begin{gathered}
l_{c h}=E \frac{G_{c}}{\left(\sigma_{\max }\right)^{2}} \\
G_{C}=\frac{N_{e} r_{e} \sin \left(\frac{\theta}{2}\right)(N)^{2}}{E}
\end{gathered}
$$

A correction of the energy release rate is obtained as a function of the radial position of the cohesive element. For both failure modes, the greater the radial distance to which the element is located, the higher the energy release rate increment.

Equations described in the previous section introduce the effect of "fiberbridging" in the cohesive model. Furthermore, a softening law is generated specifically for each of the failure modes and interfaces in the laminate. Therefore, a family of softening laws is proposed for the curved zone of each laminate.
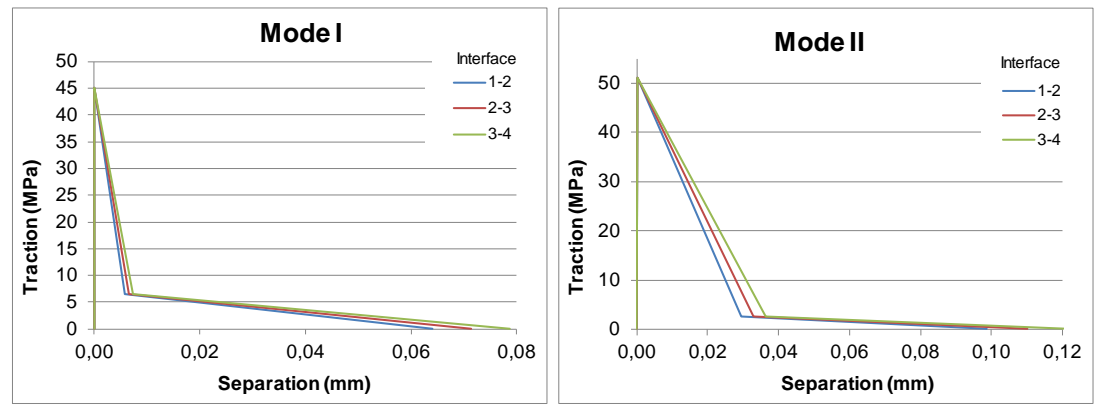
Figure 7. CB-CZM family softening laws mode I and II for the 4-layer specimens.
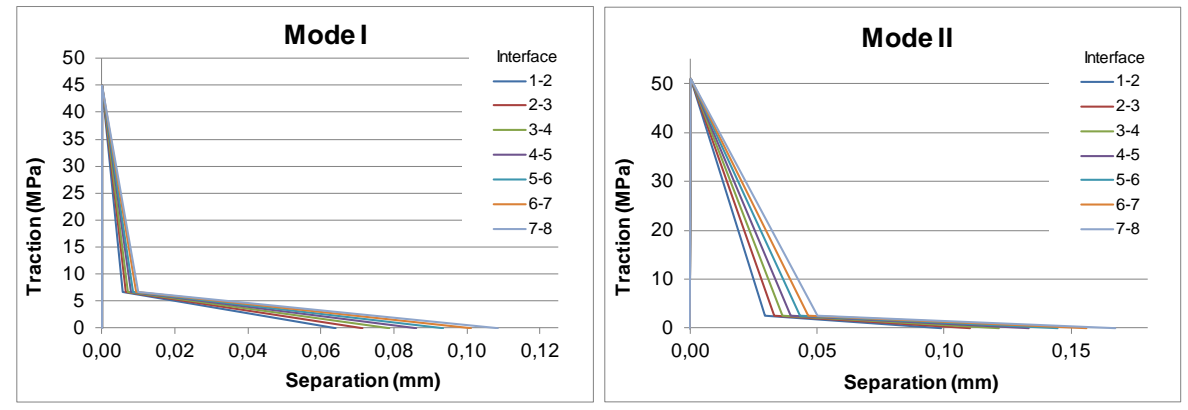

Figure 8. CB-CZM family softening laws mode I and II for the 8-layer specimens.
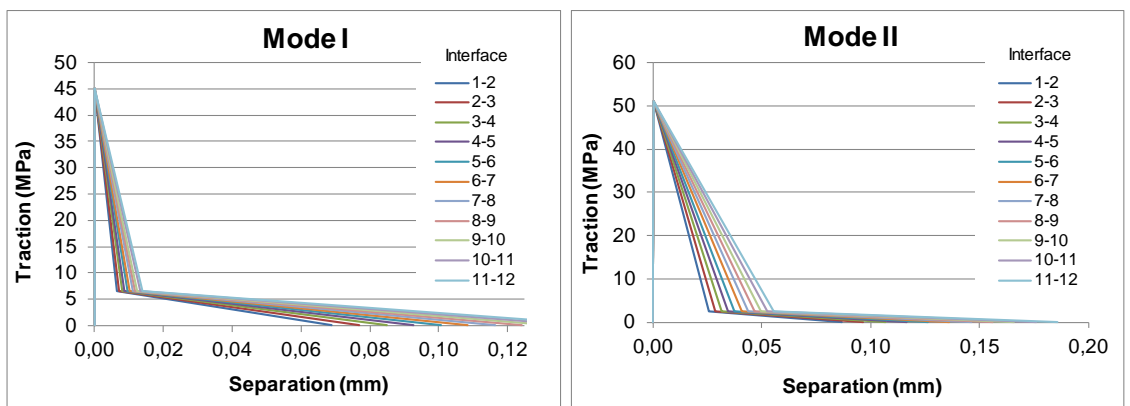

Figure 9. CB-CZM family softening laws mode I and II for the 12-layer specimens.

Figure 7 to Figure 9 show these new families of softening laws, which governs the nonlinear behaviour, for the curved zones of 4, 8 and 12 layer laminates. In all cases, it can be seen that the correction affects to a greater extent the part of the law related to "fiber-bridging" effect for mode I and cohesive degradation for mode II. This conclusion is consistent with the previous section.

\subsection{Summary of the cohesive model adapted to curved zones}

Both considerations included in the cohesive model adapted to curved zones give rise to families of softening laws that include the "fiber-bridging" phenomenon, depending on the number of interfaces existing in the curved zone laminate and the radial position of each of these interfaces. The application of the improved constitutive model for curved beams is shown in Figure 10. 


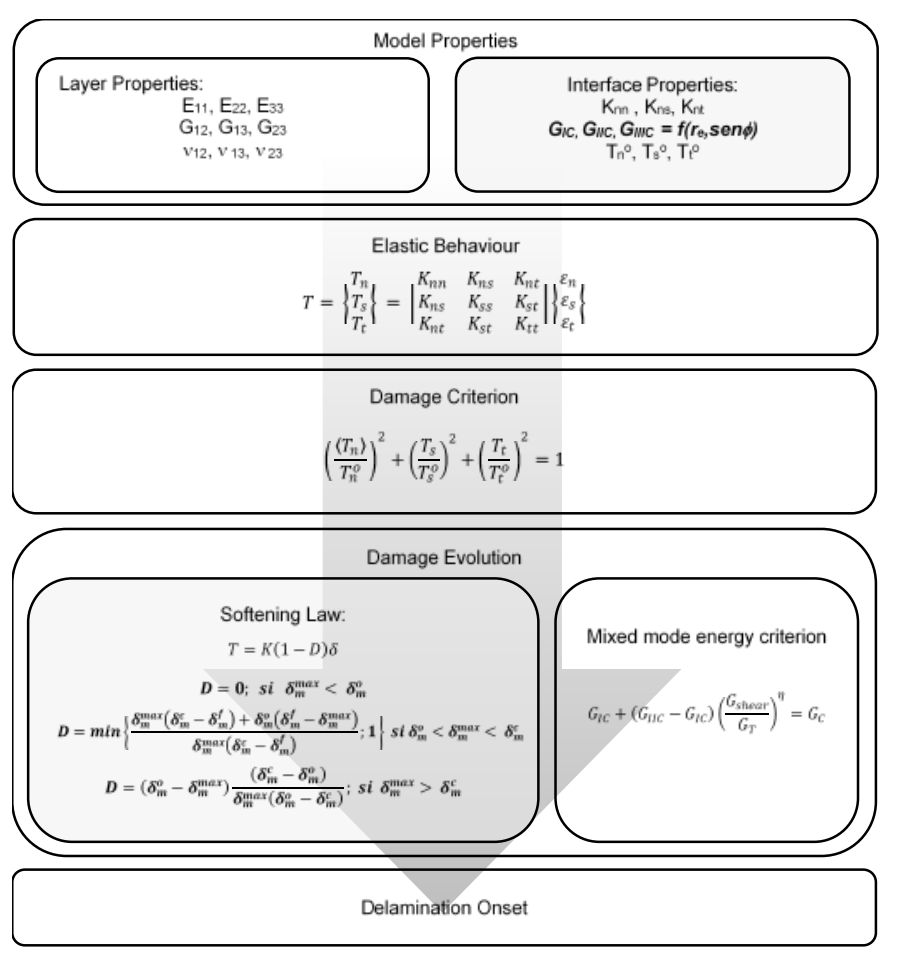

Figure 10. Enhanced constitutive model for curved beams (CB-CZM).

\section{Finite element models}

The geometric models used were the same as in the study conducted by Ranz et al [19]. Their dimensions are those shown in Figure 11 (left), as specified in Regulation ASTM D6415 to perform this kind of analysis. Models were developed in order to be compared with experimental work previously carried out [28]. To achieve this goal, models for 4, 8 and 12 layers samples were meshed and calculated.

Finite element mesh used by Ranz et al [19] utilizes a higher density of elements in the curved zone of the specimen, where delamination triggers and propagates. Each layer is discretized with 3 continuous elements through the thickness: Whereas duplicated cohesive elements are introduced between each of these layers simulating the interface.

There are multiple connecting strategies [31] in order to join cohesive and continuum elements when setting up the model. However, in the current work the 
selected one consisted of creating equivalence or coincident nodes between interface and continuum element nodes. Once cohesive elements are fully degraded such elements are removed from calculation. That allows avoiding any unwilling interference dealing to result deviations.

The computational cost increase due to the cohesive element duplication was quantified for 4 and 12 layers curved beams. Figure 11 shows a significant increase in computational cost, such cost in this particual case might be in percentage terms but it is quite low in absolute terms. 12 layers models are running in less than 20 minutes with 4 CPUS under Windows OS.

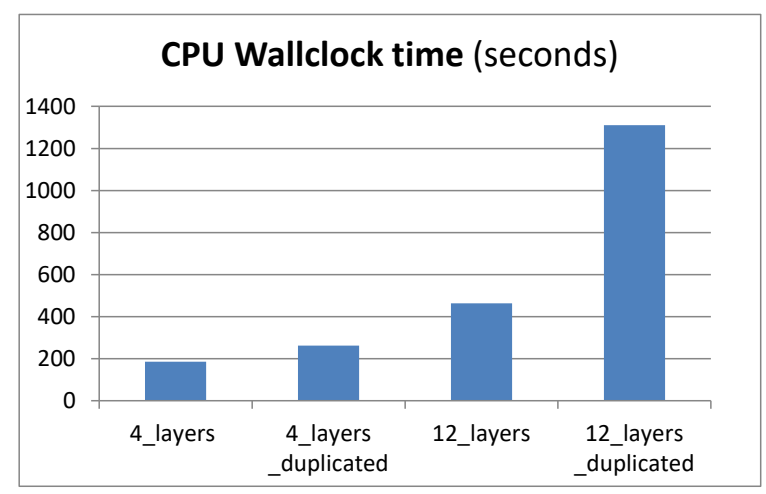

Figure 11. Computational cost of element duplication technique (4 and 12 layers).

Load rollers were set to a constant speed of $0.5 \mathrm{~mm} / \mathrm{min}$, whereas displacement on support rollers was restricted, as stated in the ASTM standard [34]. Support rollers are allowed to rotate. Friction is almost neglected between rollers and carbon sample. Initial calculations had shown no effect on results.
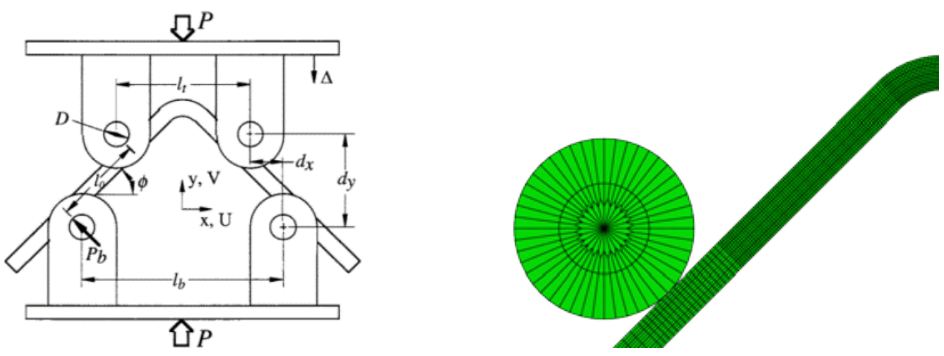
Figure 12. left) ASTM D6415 Test set-up; right) Detail of Finite Element Model (4 layers).

In the previous work where bilinear CZM [19] was applied, it was shown that the results predicted by the two-dimensional models do not show important differences with the three-dimensional ones. Therefore, two-dimensional models have been used for the application of the CB-CZM model. Elements CPE4I type were applied to model layers; while duplicated linear cohesive elements COH2D4 type have been used for the interface. These categories of elements are available in ABAQUS libraries [31].

Table 3. Properties of UD carbon-epoxy [19].

\begin{tabular}{|c|c|c|c|c|c|c|c|c|c|}
\hline \multicolumn{5}{|c|}{ Elastic Properties } & \multicolumn{5}{|c|}{ Strength Properties } \\
\hline$\overline{E_{1}(G P a)} 122$ & $\mathbf{G}_{12}(G P a)$ & 4.4 & $\begin{array}{ll}v_{12} & 0.36\end{array}$ & $X_{t}(\mathrm{MPa})$ & 1457 & $\varepsilon_{1}(\%)$ & 1.28 & $S_{12}(M P a)$ & 51.8 \\
\hline$E_{2}(G P a) \quad 8.9$ & $\mathbf{G}_{13}(\mathrm{GPa})$ & 4.4 & $v_{13} * 0.18$ & $Y_{t}(\mathrm{MPa})$ & 28.5 & $\varepsilon_{2}(\%)$ & 0.36 & $\gamma_{12}(\%)$ & 2.97 \\
\hline$E_{3}(G P a)^{*} 8.9$ & $\mathbf{G}_{23}(\mathrm{GPa})$ & 3.2 & $v_{23} * 0.36$ & $X_{c}(\mathrm{MPa})$ & 775.3 & $\varepsilon_{1 c}(\%)$ & 0.67 & $\boldsymbol{S}_{13}(\mathrm{MPa})$ & 51.2 \\
\hline \multicolumn{4}{|c|}{ Fiber volume $V_{f}(\%) 56$} & $Y_{c}(M P a)$ & 113.6 & $\varepsilon_{2 \mathrm{c}}(\%)$ & 2.01 & $S_{23}(\mathrm{MPa})$ & * 51.2 \\
\hline
\end{tabular}

When the CZM is implemented in a finite element model, the material description is separated into the material properties related to the laminate (Table 3) and into the constitutive model properties of the cohesive surface. In the case of interface properties, a normal stiffness $\left(\mathrm{K}_{\mathrm{n}}\right)$ of $4.6 \times 10^{5} \mathrm{~N} / \mathrm{mm}^{3}$ and a shear stiffness $\left(\mathrm{K}_{\mathrm{s}}\right)$ of $2.3 \times 10^{5} \mathrm{~N} / \mathrm{mm}^{3}$ are used. The interface strength (T) and the critical energy release rate $\left(G_{c}\right)$ are defined by the families of softening laws of the CB-CZM described in the previous section (Figure 7 to Figure 9), according to the predominant failure mode.

Definition of superposition laws was implemented via equation 1 . This is implemented via calculation through ABAQUS cohesive section definition. Values corresponding to softening laws are shown in Figure 5 (for a single layer) and Figure 7, 8 and 9 (for 4, 8 and 12 layers, respectively). 


\section{Results and discussion}

In this section, numerical results obtained from the cohesive model adapted to curved zones (CB-CMZ) are shown. Unidirectional specimens including 4, 8 and 12 layers were analysed. The different graphs of normalized force versus displacement, obtained from numerical simulation, are integrated with the evolution of the interlaminar normal stress $\left(\mathrm{S}_{33}\right)$. Finally, they are compared with the experimental results and those obtained with the bilinear model previously used by Ranz [19], as well as with the values of Curved Beam Strength (CBS) and Interlaminar Tensile Strength (ILTS).

\subsection{Results obtained by applying $C B-C Z M$ on UD laminates}

The evolution of interlaminar normal stress $\left(\mathrm{S}_{33}\right)$ for the case of 4-layer specimen numerically simulated by applying the adapted model, CB-CZM, is shown in Figure 13. As in the bilinear model, a linear evolution of the force/displacement curve is observed at the beginning of the virtual test until a maximum force of approximately $44 \mathrm{~N} / \mathrm{mm}$ is reached, which corresponds to a displacement of $5 \mathrm{~mm}$. Just a moment before reaching that force, the maximum interface strength has also been reached, calculated by the failure quadratic criterion, which has been implemented for cohesive elements. It is at that point when the cohesive elements that introduce the cohesive law begin to degrade, quickly reaching the critical energy release rate and giving rise to the failure of those elements. The delamination triggers between the $2^{\text {nd }}$ and $3^{\text {rd }}$ laminate layers. However, degraded cohesive elements introduce the "fiber-bridging" phenomenon. Therefore, they begin to work supporting a sustained force and reaching a high level of separation between the corresponding interface faces, preventing the force supported from collapsing. In this way, the supported load falls to a value of $29 \mathrm{~N} / \mathrm{mm}$, higher than the fall that happened for the case of the bilinear model (Figure 16). 


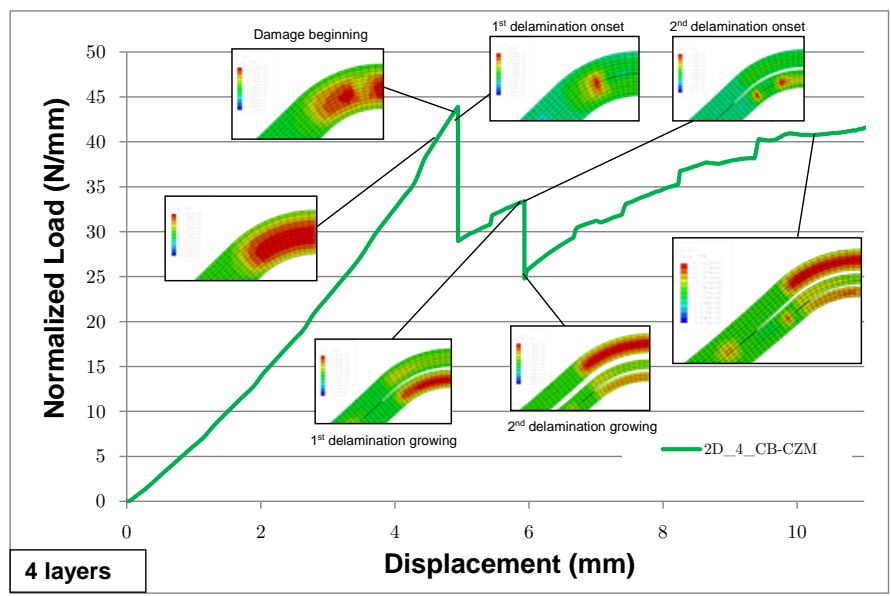

Figure 13. $\mathrm{S}_{33}$ distribution along the force-displacement graph (4 layers).

Subsequently, a redistribution of stress takes place in the laminate as delamination progresses. Therefore, both regions between which the delamination has occurred begin to work independently. Besides, the separation between them is progressively increased and this fact leads to the failure of cohesive elements in which the "fiber-bridging" effect happened. The supported force is recovered until reaching a load level of $34 \mathrm{~N} / \mathrm{mm}$, related to a $6 \mathrm{~mm}$ displacement. At that moment, cohesive elements located between first and second layer also reach their interface strength and begin to degrade. This effect causes a second delamination and a force drop to 25 $\mathrm{N} / \mathrm{mm}$. Thereafter, the load supported by the sample is increased until the final failure.

The evolution of interlaminar normal stress $\left(\mathrm{S}_{33}\right)$ for the case of 8-layer unidirectional specimen numerically simulated by applying the adapted model, CBCZM, is shown in Figure 14. A maximum force of $66 \mathrm{~N} / \mathrm{mm}$ is reached for $2 \mathrm{~mm}$ of displacement. At that time, the quadratic failure criterion defined for the cohesive elements is satisfied and they begin to degrade. They quickly reach the critical energy release rate and their failure occurs. Therefore, the first delamination triggers between the third and fourth laminate layers. However, unlike the bilinear model, delamination 
progress is stopped by the contribution of "fiber-bridging" effect in the cohesive elements. According to this phenomenon a redistribution of efforts occurs, provoking a second delamination to appear between the fourth and fifth layers. This second delamination evolves more quickly, until reaching a force drop that stops at $48 \mathrm{~N} / \mathrm{mm}$. From that moment, a force recovery is produced up to a value of $58 \mathrm{~N} / \mathrm{mm}$ for a displacement of $2.8 \mathrm{~mm}$. At that point, the cohesive elements that simulate the interface between the second and third layers reach their cohesive strength and begin the subsequent degradation and final failure, producing the delamination between these two layers. When this new delamination starts, a sudden fall in the force is observed, due to the delamination onset between the first and second layers. At the moment when a load of $44 \mathrm{~N} / \mathrm{mm}$ is reached, the force starts to recover until reaching a load value of 69 $\mathrm{N} / \mathrm{mm}$, for a displacement of $5.4 \mathrm{~mm}$. Then, a fifth delamination starts between the sixth and seventh layers, which causes a new drop in the supported load. The presence of cohesive elements with "fiber-bridging" phenomenon is shown in the last image of Figure 14. Those elements preserve the tie between adjacent layers in which the interface has failed in cohesive terms.

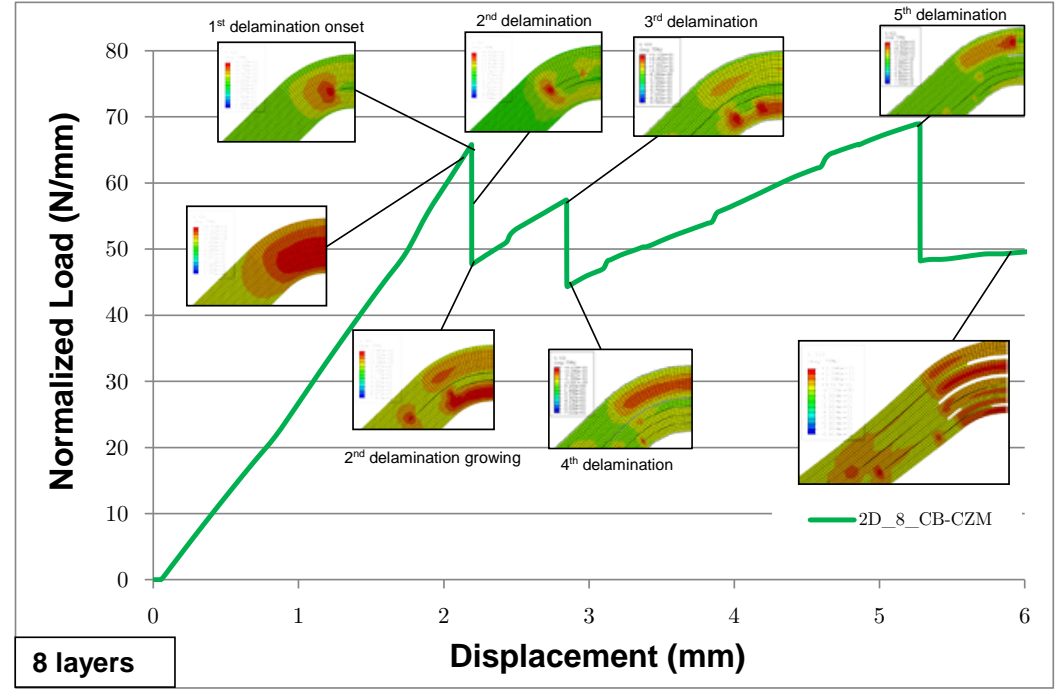

Figure 14. $\mathrm{S}_{33}$ distribution along the force-displacement graph (8 layers). 
The evolution of interlaminar normal stress $\left(\mathrm{S}_{33}\right)$ for the case of 12-layer unidirectional specimen numerically simulated is shown in Figure 14. Identically to the bilinear model, a linear evolution of the force-displacement curve is initially observed, when reaching a maximum force of approximately $112 \mathrm{~N} / \mathrm{mm}$, which corresponds to a displacement of $1.6 \mathrm{~mm}$. At that time, the cohesive elements begin to degrade and quickly reach the critical energy release rate, initiating their progressive failure. First delamination takes place between fourth and fifth laminate layers. Therefore, the bearing capacity of the test sample drops and the second delamination onset between the fifth and sixth layers occurs at once. This second delamination evolves more promptly than the previous one, until reaching a loading level of $79 \mathrm{~N} / \mathrm{mm}$. From that point, a recovery of the supported force come about until a force of $110 \mathrm{~N} / \mathrm{mm}$ is reached, which corresponds to a displacement of $2.5 \mathrm{~mm}$. This new fall is due to the start of the third delamination between the second and third layers. In the case of the bilinear model, this fall occurred for a displacement of $3.5 \mathrm{~mm}$. This fall reaches a value of $98 \mathrm{~N} / \mathrm{mm}$, but recovers quickly until reaching a maximum force of $125 \mathrm{~N} / \mathrm{mm}$, for a displacement of $3.8 \mathrm{~mm}$, where the failure of cohesive elements between the eighth and ninth layers starts. This last delamination generates a redistribution of stresses in the laminate, causing the delamination between the ninth and tenth layers throughout this discharge process, falling force to $78 \mathrm{~N} / \mathrm{mm}$, and subsequently producing a maintained force supported. 


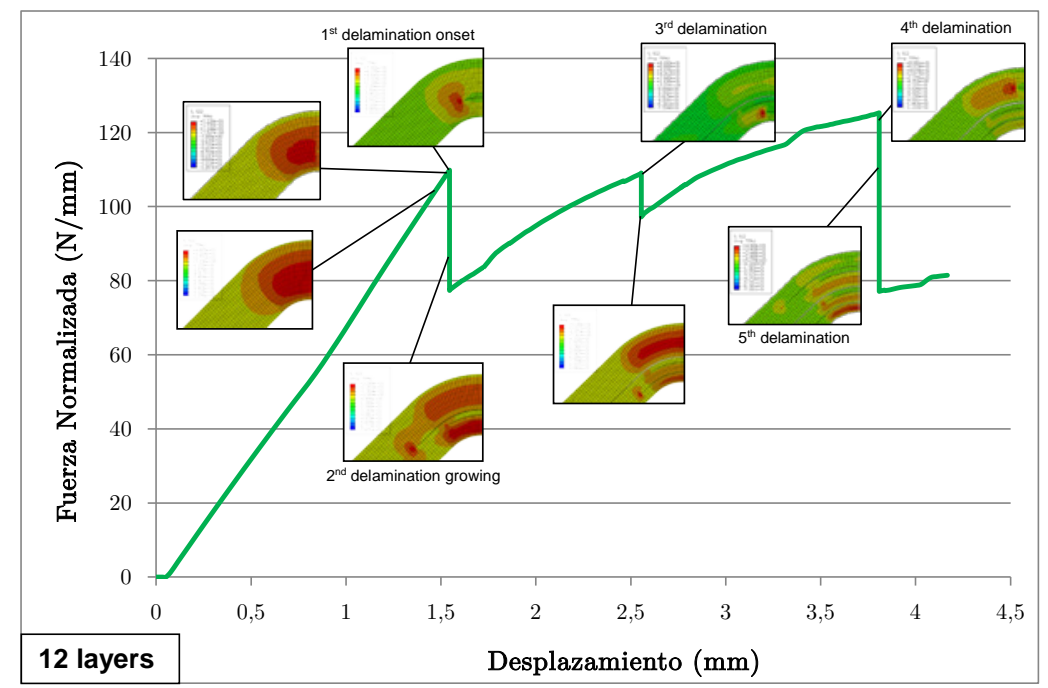

Figure 15. $\mathrm{S}_{33}$ distribution along the force-displacement graph (12 layers).

\subsection{Numerical-experimental model correlation for $C B-C Z M$}

Results of the bilinear model (red) and the CB-CZM model (green) are compared in Figure 16 to Figure 18 with those obtained experimentally (black) by real tests for 4, 8 and 12 layer samples. Stiffness and the maximum force reached employing both models are identical in all cases. Numerical strength predictions are very close to those obtained experimentally.

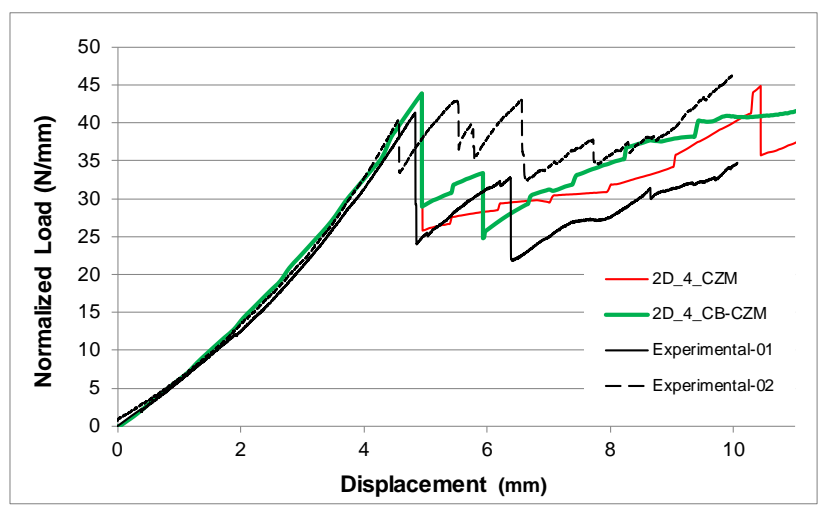

Figure 16. Correlation of load vs displacement curves for 4 layer coupons. 


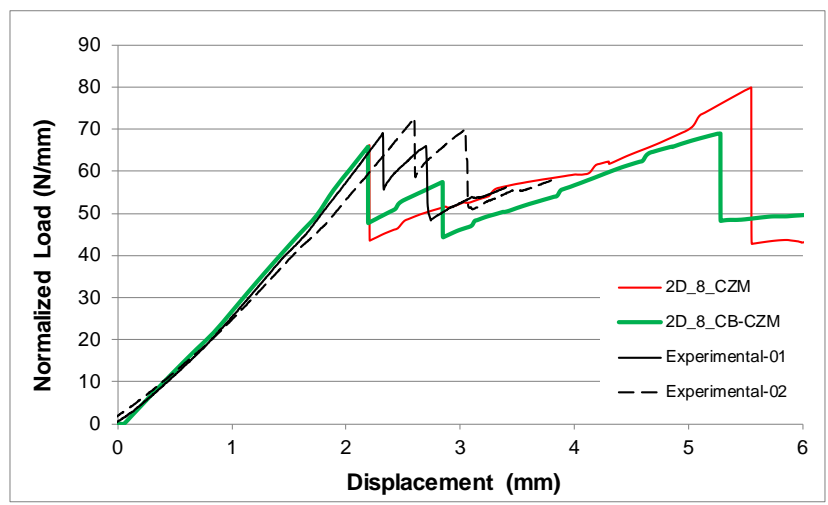

Figure 17. Correlation of load vs displacement curves for 8 layer coupons.

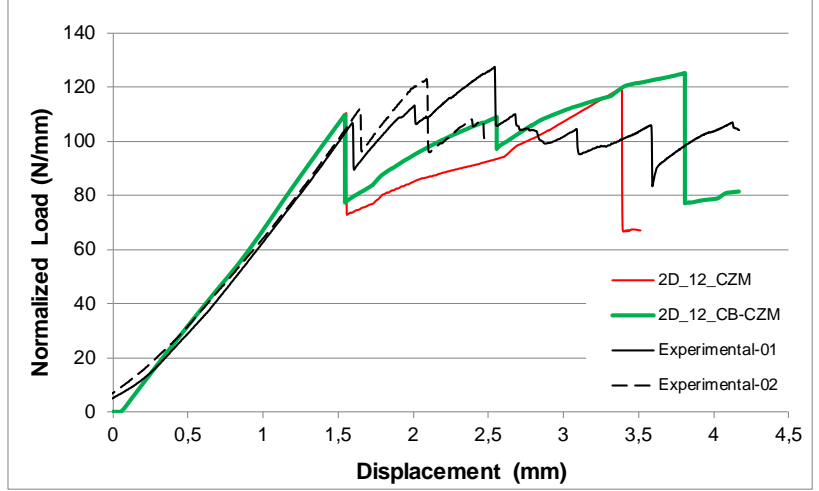

Figure 18. Correlation of load vs displacement curves for 12 layer coupons.

The most significant differences are related to post-failure behavior, once the first delamination has started. Thanks to the new considerations included in the CB-CZM model, results are closer to those obtained experimentally, as can be seen in the figures and collected in Table 4. On the one hand, the force drop that occurs after the first delamination is more limited in the graphs of the CB-CZM model, as in the tests. On the other hand, the subsequent drops in force obtained by this model after first delamination, take place with lower displacement, as occurs in the real tests. In addition, it is appreciated that the load recovery presents the same tendency between the numerical and experimental models. 
Table 4. Post failure values of significative loads and position.

\begin{tabular}{lccccccccc}
\hline & \multicolumn{3}{c}{4 layers } & \multicolumn{3}{c}{8 layers } & \multicolumn{3}{c}{12 layers } \\
\cline { 2 - 9 } & Exp. & CZM & CB-CZM & Exp. & CZM & CB-CZM & Exp. & CZM & CB-CZM \\
\hline \hline $1^{\text {st }}$ Load Drop (N/mm) & 24 & 26 & 29 & 55 & 43 & 48 & 89 & 73 & 79 \\
\hline \hline $2^{\text {nd }}$ Max Load (N/mm) & 33 & 44 & 34 & 64 & 80 & 58 & 127 & 118 & 110 \\
\hline \hline $2^{\text {na }}$ Max Load position (mm) & 6.4 & 10.4 & 5.9 & 2.7 & 5.5 & 2.8 & 2.5 & 3.4 & 2.5 \\
\hline \hline $2^{\text {na }}$ Load Drop (N/mm) & 23 & 36 & 25 & 48 & 42 & 44 & 106 & 66 & 98 \\
\hline
\end{tabular}

Figure 19 shows the evolution of CBS (left) and ILTS (right), as long as the applied displacement. CBS and ILTS were obtained by means of the bilinear model (dashed curves) and the CB-CZM model (continuous curves). CBS and ILTS values calculated by both models are identical at the time of the first delamination. The behaviour of the test specimens with different number of layers, up to the first delamination takes place, is also the same in both cohesive models. The main differences, as in the force-displacement graphs shown above, arise in the post-failure behaviour for both models.

The main conclusion drawn from this analysis is that the adapted model CBCZM provides the closest results to the experimental ones.
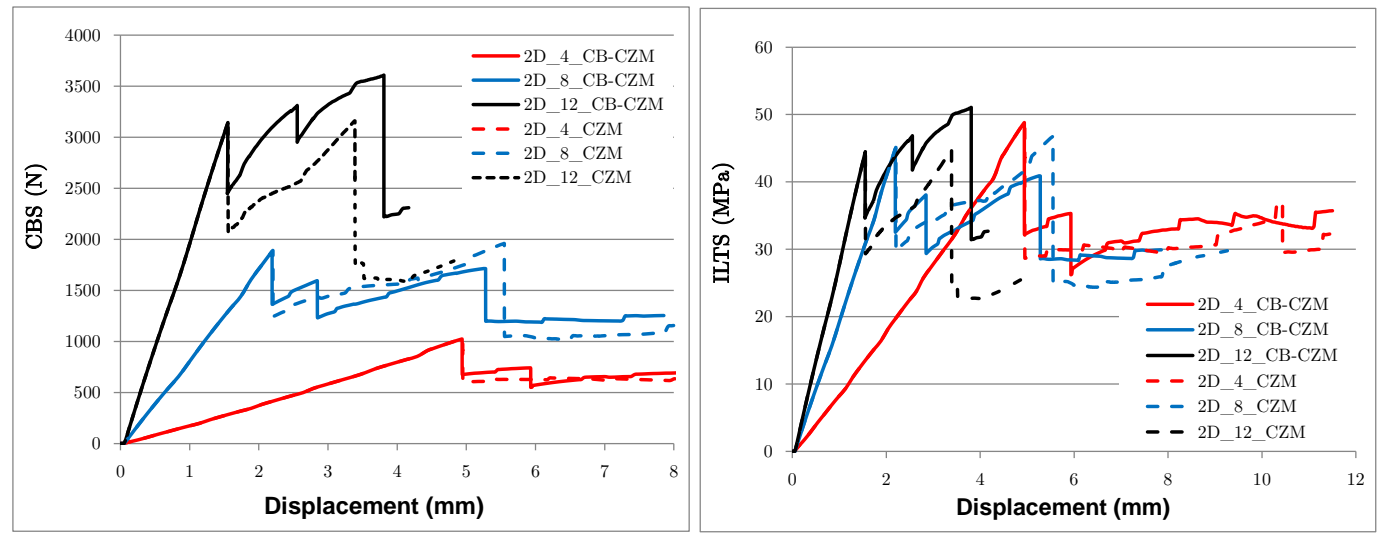

Figure 19. CZM vs CB-CZM: CBS (left); ILTS (right). 


\section{Conclusions}

The new adapted Cohesive Zone Model for Curved Beams (CB-CZM) proposed in this paper, includes considerations aimed at improving the behavior prediction of unidirectional composite materials, once the first delamination has begun. On the one hand, "fiber-bridging" phenomenon was included through the implementation of two different laws, which were applied by the introduction of a double layer of cohesive elements. On the other hand, the critical deformation energy release rate was adapted to the radial position of the cohesive elements in order to avoid numerical problems.

Thanks to the new considerations included in the new CB-CZM model, obtained results are closer to those obtained experimentally. First, the force drop that occurs after the initial delamination onset is more limited in the graphs obtained by this model, as it happens in the real tests. Second, the subsequent force drops that occur after the first delamination are related to smaller displacements in the CB-CZM model, as occurs in the real behavior of UD composite specimens. Furthermore, the load recovery presents the same trend in numerical models and experimental tests.

\section{References}

[1] W. Hao, D. Ge, Y. Ma, X. Yao, and Y. Shi, "Experimental investigation on deformation and strength of carbon/epoxy laminated curved beams," Polym. Test., vol. 31, no. 4, pp. 520-526, 2012, doi: 10.1016/j.polymertesting.2012.02.003.

[2] Raju, "Delamination damage analysis of curved composites subjected to compressive load using cohesive zone modelling,” 2014.

[3] W. Cui, T. Liu, J. Len, and R. Ruo, “Interlaminar tensile strength (ILTS) measurement of woven glass/polyester laminates using four-point curved beam specimen,” Compos. Part A Appl. Sci. Manuf., vol. 27, no. 11, pp. 1097-1105, 1996, doi: 10.1016/1359-835X(96)00071-1. 
[4] S. C. Avalon and S. L. Donaldson, "Strength of composite angle brackets with multiple geometries and nanofiber-enhanced resins,” J. Compos. Mater., vol. 45, no. 9, pp. 1017-1030, 2011, doi: 10.1177/0021998310381538.

[5] R. Martin, "Delamination Failure in a Unidirectional Curved Composite Laminate,” Compos. Mater. Test. Des. (Tenth Vol., pp. 365-365-19, 2009, doi: 10.1520/stp20170s.

[6] R. Roos, G. Kress, M. Barbezat, and P. Ermanni, "Enhanced model for interlaminar normal stress in singly curved laminates,” Compos. Struct., vol. 80, no. 3, pp. 327-333, 2007, doi: 10.1016/j.compstruct.2006.05.022.

[7] B. Gozluklu, I. Uyar, and D. Coker, "Intersonic delamination in curved thick composite laminates under quasi-static loading,” Mech. Mater., vol. 80, no. PB, pp. 163-182, 2015, doi: 10.1016/j.mechmat.2014.07.013.

[8] J.-H. Kim, K.-H. Nguyen, J.-H. Choi, and J.-H. Kweon, "Experimental and finite element analysis of curved composite structures with C-section,” Compos. Struct., vol. 140, pp. 106-117, 2016, doi: 10.1016/j.compstruct.2015.12.048.

[9] H. Ju, K.-H. Nguyen, S.-S. Chae, and J.-H. Kweon, “Delamination strength of composite curved beams reinforced by grooved stainless-steel Z-pins,” Compos. Struct., vol. 180, pp. 497-506, 2017, doi: 10.1016/j.compstruct.2017.08.018.

[10] K. H. Nguyen, H. W. Ju, V. H. Truong, and J. H. Kweon, "Delamination analysis of multi-angle composite curved beams using an out-of-autoclave material,” Compos. Struct., vol. 183, no. 1, pp. 320-330, 2017, doi: 10.1016/j.compstruct.2017.03.078.

[11] Q. Yang and B. Cox, “Cohesive models for damage evolution in laminated composites,” Int. J. Fract., vol. 133, no. 2, pp. 107-137, 2005, doi: 10.1007/s10704-005-4729-6.

[12] M. Elices, G. V. Guinea, J. Gómez, and J. Planas, “The cohesive zone model: Advantages, limitations and challenges,” Eng. Fract. Mech., vol. 69, no. 2, pp. 137-163, 2001, doi: 10.1016/S0013-7944(01)00083-2.

[13] P. W. Harper and S. R. Hallett, "Cohesive zone length in numerical simulations of composite delamination,” Eng. Fract. Mech., vol. 75, no. 16, pp. 4774-4792, 2008, doi: 10.1016/j.engfracmech.2008.06.004.

[14] A. Turon, C. G. Dávila, P. P. Camanho, and J. Costa, “An Engineering Solution for using Coarse Meshes in the Simulation of Delamination With Cohesive Zone Models,” Nasa/Tm-2005-213547, no. March, pp. 1-26, 2005. 
[15] G. Alfano, "On the influence of the shape of the interface law on the application of cohesive-zone models,” Compos. Sci. Technol., vol. 66, no. 6, pp. 723-730, 2006, doi: 10.1016/j.compscitech.2004.12.024.

[16] P. P. Camanho, C. G. Dávila, and M. F. De Moura, “Numerical simulation of mixed-mode progressive delamination in composite materials,” J. Compos. Mater., vol. 37, no. 16, pp. 1415-1438, 2003, doi: 10.1177/0021998303034505.

[17] G. Alfano and M. A. Crisfield, "Finite element interface models for the delamination analysis of laminated composites: Mechanical and computational issues,” Int. J. Numer. Methods Eng., vol. 50, no. 7, pp. 1701-1736, 2001, doi: 10.1002/nme.93.

[18] S. T. Pinho, L. Iannucci, and P. Robinson, "Formulation and implementation of decohesion elements in an explicit finite element code," Compos. Part A Appl. Sci. Manuf., vol. 37, no. 5, pp. 778-789, 2006, doi:

10.1016/j.compositesa.2005.06.007.

[19] D. Ranz, J. Cuartero, L. Castejon, R. Miralbes, and H. Malon, “A cohesive zone model approach to interlaminar behaviour of carbon/epoxy laminated curved beams,” Compos. Struct., vol. 238, p. 111983, Apr. 2020, doi:

10.1016/j.compstruct.2020.111983.

[20] S. S. Rudraraju, K. Garikipati, A. M. Waas, and B. A. Bednarcyk, “On the Theory and Numerical Simulation of Cohesive Crack Propagation With Application to Fiber-Reinforced Composites,” 2013.

[21] P. P. Camanho, C. G. Davila, and C. A. Rose, “A procedure for superposing linear cohesive laws to represent multiple damage mechanisms in the fracture of composites of composites,” Int. J. Fract., pp. 211-223, 2009, doi: 10.1007/s10704-009-9366-z.

[22] M. Saeedifar, M. Fotouhi, M. Ahmadi Najafabadi, and H. Hosseini Toudeshky, "Prediction of delamination growth in laminated composites using acoustic emission and Cohesive Zone Modeling techniques,” Compos. Struct., vol. 124, pp. 120-127, Jun. 2015, doi: 10.1016/j.compstruct.2015.01.003.

[23] S. Li, J. A. Schroeder, M. D. Thouless, P. D. Zavattieri, and A. M. Waas, "Use of mode-I cohesive-zone models to describe the fracture of an adhesivelybonded polymer-matrix composite,” Compos. Sci. Technol., vol. 65, no. 2, pp. 281-293, 2004, doi: 10.1016/j.compscitech.2004.07.009. 
[24] S. Jacques, I. De Baere, and W. Van Paepegem, “Analysis of the Numerical and Geometrical Parameters Influencing the Simulation of Mode I and Mode II Delamination Growth in Unidirectional and Textile Composites,” Appl. Compos. Mater., vol. 22, no. 6, pp. 637-668, 2015, doi: 10.1007/s10443-014-9429-9.

[25] A. Turon, C. G. Dávila, P. P. Camanho, and J. Costa, “An Engineering Solution for Solving Mesh Size Effects in the Simulation of Delamination with Cohesive Zone Models,” Nasa Langley Res. Cent., vol. TM, no. June 2005, 2005.

[26] K. Song, C. G. Dávila, and C. A. Rose, "Guidelines and parameter selection for the simulation of progressive delamination,” 2008 ABAQUS User's Conf., pp. 1-15, 2008.

[27] C. G. Hoover and Z. P. Bažant, “Cohesive crack, size effect, crack band and work-of-fracture models compared to comprehensive concrete fracture tests,” Int. J. Fract., vol. 187, no. 1, pp. 133-143, 2014, doi: 10.1007/s10704-0139926-0.

[28] D. Ranz, J. Cuartero, A. Miravete, and R. Miralbes, "Experimental research into interlaminar tensile strength of carbon/epoxy laminated curved beams,” Compos. Struct., vol. 164, pp. 189-197, Mar. 2017, doi: 10.1016/j.compstruct.2016.12.010.

[29] M. Kenane and M. L. Benzeggagh, "Measurement of Mixed-Mode delamination Fracture Toughness of unidirectional Glass/Epoxy Composites With MixedMode Bending Apparatus,” Compos. Sci. Technol., vol. 49, pp. 439-449, 1996.

[30] S. Li, M. D. Thouless, A. M. Waas, J. A. Schroeder, and P. D. Zavattieri, "Mixed-mode cohesive-zone models for fracture of an adhesively bonded polymer-matrix composite,” Eng. Fract. Mech., vol. 73, no. 1, pp. 64-78, 2006, doi: 10.1016/j.engfracmech.2005.07.004.

[31] (C) Dassault Systèmes, “Abaqus 6.11 Online Documentation.” [Online]. Available: http://130.149.89.49:2080/v6.11/books/usb/default.htm?startat=pt04ch17s01aus 101.html. [Accessed: 05-Apr-2019].

[32] N. Moes and B. Ted, "Extended finite element method for cohesive crack growth,” Eng. Fract. Mech., vol. 69, no. June, pp. 813-833, 2002, doi: 10.1016/S0013-7944(01)00128-X. 
[33] M. L. Falk, A. Needleman, and J. R. Rice, “A critical evaluation of cohesive zone models of dynamic fractur,” Le J. Phys. IV, vol. 11, no. PR5, pp. Pr5-43Pr5-50, 2007, doi: 10.1051/jp4:2001506.

[34] ASTM, “ASTM D6415/D6425M-06a Standard Test Method for Measuring the Curved Beam Strength of a Fiber-Reinforced Polymer Matrix Composite,” Annu. B. ASTM Stand., vol. 15.03, no. Reapproved 2013, pp. 1-10, 2015, doi: 10.1520/D6415.

The published version of this article: Enhanced cohesive zone model to predict delamination behavior of carbon/epoxy laminated curved beams, can be found at Mechanics of Advanced Materials and Structures. DOI: $\underline{\text { https://doi.org/10.1080/15376494.2020.1769232 }}$ 